\title{
Objective cough frequency monitoring in real-world practice
}

\author{
Ji-Hyang Lee ${ }^{1}$, Woo-Jung Song $\mathbb{B}^{1}$, Eva Millqvist ${ }^{2}$ and Alyn H. Morice $\mathbb{B}^{3}$
}

${ }^{1}$ Dept of Allergy and Clinical Immunology, Asan Medical Center, University of Ulsan College of Medicine, Seoul, Korea. ${ }^{2}$ Dept of Internal Medicine/Respiratory Medicine and Allergology, Sahlgrenska University Hospital, University of Gothenburg, Gothenburg, Sweden. ${ }^{3}$ Respiratory Medicine, Institute for Clinical and Applied Health Research, Hull York Medical School, Hull, UK.

Corresponding author: Woo-Jung Song (swj0126@gmail.com)

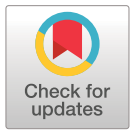

Copyright (T) The authors 2021

This version is distributed under the terms of the Creative Commons Attribution NonCommercial Licence 4.0. For commercial reproduction rights and permissions contact permissions@ersnet.org

Received: 25 Aug 2021 Accepted: 13 Sept 2021

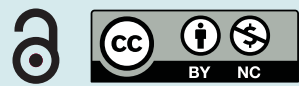

Shareable abstract (@ERSpublications)

There are possibilities and obstacles to the use of objective cough monitoring in real-world practice. Advances in technology will overcome the difficulties and continuous real-time cough monitoring will then provide a valuable tool in cough management. https://bit.ly/3lu5cfk

Cite this article as: Lee J-H, Song W-J, Millqvist E, et al. Objective cough frequency monitoring in realworld practice. ERJ Open Res 2021; 7: 00545-2021 [DOI: 10.1183/23120541.00545-2021].

For those of us seeing patients with chronic cough, there is one great advantage in comparison with other specialities dealing with recurrent symptoms such as migraine, chronic pain, and psychiatric conditions. We can measure cough.

There are three metrics available to us. Inhalational cough challenge with a variety of tussive agents has been used since the 1950s. This has given us clear evidence that cough reflex hypersensitivity is a cardinal feature of the condition. However, like the bronchial hyperreactivity seen in asthma, this is "not necessary or sufficient to make the diagnosis” to quote the 2021 Global Initiative for Asthma guidelines. Cough challenge is therefore reserved for the experimental study of the pathophysiology of the cough reflex.

Patient-reported outcomes from the simple "score your cough out of 10" used in the clinic, to validated quality of life instruments such as the Leicester Cough Questionnaire quantify the patient experience which, because of its subjective nature, captures a different facet of the condition of chronic cough [1, 2].

Finally, because cough is forced expulsive manoeuvre, usually against a closed glottis, it is associated with a characteristic sound [3]. Since the 1960s, it has been objectively recorded (see figure 1 [4]) and the fundamental elements of this assessment are essentially unchanged. A microphone captures the sound which is recorded and timed (now digitally). The difficult part, which remains unsolved, is how to convert these recordings into cough counts for continuous real-time analysis. Validated tools, such as the Leicester Cough Monitor (LCM) and VitaloJAK [5, 6], are now accepted, with 24-h recordings used as the primary end-point in trials of anti-tussive therapy [7, 8]. However, the feasibility and utility of such measures in real-world clinical practice remains to be tested.

In this issue of ERJ Open Research, VerTigan et al. [9] describe their experiences with using LCM in 174 patients and 15 controls at tertiary outpatient clinics and assess the feasibility and clinical utility of objective cough frequency monitoring. The analyses were retrospective, but reveal interesting insights into the practical application of such technology. So, should non-specialists invest in a cough recorder for their clinics?

In terms of feasibility, the majority of both patients and clinicians were positive about using the cough monitors. Approximately three quarters of patients were positive about wearing the monitoring device and could use it correctly and $93 \%$ of the recordings were interpretable. The time required for setting up equipment and patient explanation (such as checking batteries, printing instructions, testing, and explaining the instructions to the patients) was between 10 and $20 \mathrm{~min}$ in total. Since the LCM uses a computer-based algorithm to "listen" for cough and cough-like sounds, the analysis time per recording averaged a mere $10 \mathrm{~min}$, so in terms of practicality of use of the LCM seems feasible. 

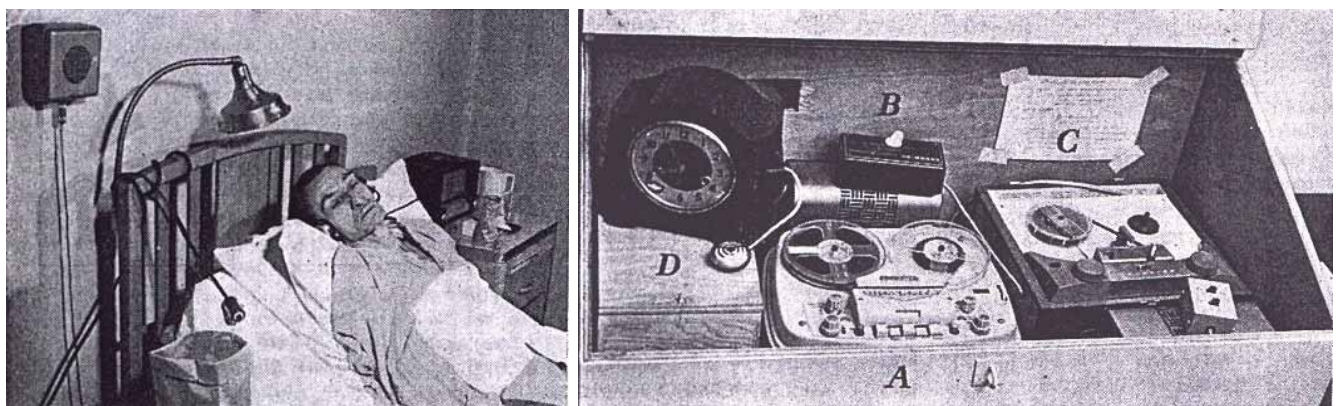

FIGURE 1 The essential equipment for cough recording. Microphone in position above the head of bed (left-hand image) and tape recorder system (right image). A: main tape recorder; B: electronic on/off unit; C: playback unit with pre-recorded voice; D: chiming clock with microphone. Reproduced from [4] with permission.

However, there is a major limitation inherent in existing cough recording technologies. All current systems record ambient sound for subsequent analysis. We have previously noted a marked reluctance in both patients and relatives to the personal intrusion of unfiltered sound recordings [10]. This is confirmed by the present study in that a quarter of the patients would not agree to carry the device. In an ongoing cough treatment study, 30 chronic cough patients wore the LCM on several occasions without major complaint, but all had meticulous information and instruction about the purpose, use and personal integrity of the recordings (unpublished data; Eva Millqvist). Until such issues of privacy and confidentiality are solved, such as by real-time analysis within the device and the subsequent logging of cough sounds alone, then widespread adoption outside of clinical trials is unlikely.

The authors claim that 24-h cough monitoring was valuable for speech pathologists in planning individual treatments based on a temporal pattern of cough frequency. However, such evaluation requires considerable experience. In their figure 2, they present a "typical” hourly breakdown of cough recording. It shows more or less continual, but intermittent coughing over the whole day. This is a most unusual finding since cough typically diminishes when the patient is asleep to $\sim 10 \%$ of the awake cough frequency. Indeed, this observation is confirmed by their own data comparing daytime and night-time cough (see table 4 in VertigAn et al. [9]).

Furthermore, they claim that cough frequency data is useful for clinicians in evaluating therapeutic responses. However, these were uncontrolled observations and the experience in recent large randomised controlled trials using 24-h cough monitoring is that placebo responses are observed in up to 50\% of patients [11], a similar figure to that observed by VeRTigan et al. [9].

Perhaps the most interesting observation was the inclusion of 50 patients with inducible laryngeal obstruction and 21 with a diagnosis of severe asthma. These patient groups exhibited cough counts which were very similar to their patients characterised as refractory chronic cough. The authors come to the surprising conclusion that "cough is increased not only in chronic refractory cough but in other diseases". If one looks at the demographics of these other patients, they are predominantly middle-aged women in their sixth decade and three quarters are female (table 1 in VerTigan et al. [9]). This is exactly the characteristics described in over 10000 patients presenting to specialist cough clinics worldwide [12] Given this remarkable similarity is it not more likely that these "other diseases" in fact represent different presentations of the underlying diagnosis of refractory chronic cough?

In support of this hypothesis, patients were administered the Laryngeal Hypersensitivity Questionnaire (LHQ) with the majority scoring outside the established normal range. The LHQ shares several questions with the Hull Airways Reflux Questionnaire (HARQ) which was administered to nearly 2000 patients with refractory or unexplained chronic cough, 95\% of whom scored above the upper limit of normal [13]. The HARQ was designed around the known pathophysiology of extra-oesophageal or airway reflux, suggesting a common aetiology may underlie coughing in these diagnoses.

In summary, Vertigan et al. [9] analysed the feasibility of LCM and identified several possibilities and obstacles to its use in real-world practice. Advances in cough assessments and technology will, in future, 
overcome these difficulties and continuous real time cough monitoring will then provide a valuable tool in diagnosis and management, whatever label is placed on our patients who suffer greatly from the disease of chronic cough.

Provenance: Commissioned article, peer reviewed.

Conflict of interest: J-H. Lee has no conflict of interest to declare. W-J. Song reports grants from MSD and AstraZeneca; consulting fees from MSD and AstraZeneca; and lecture fees from MSD, AstraZeneca, GlaxoSmithKline (GSK) and Novartis. E. Millqvist has no conflict of interest to declare. A.H. Morice has received consulting fees from Bayer, Bellus, Boehringer Ingelheim, Merck, Pfizer, Proctor \& Gamble and Shionogi; lecture fees from Boehringer Ingelheim and AstraZeneca; and grant support from Proctor \& Gamble, Merck, Afferent and Infirst.

\section{References}

1 Birring SS, Prudon B, Carr AJ, et al. Development of a symptom specific health status measure for patients with chronic cough: Leicester Cough Questionnaire (LCQ). Thorax 2003; 58: 339-343.

2 Morice AH, Millqvist E, Bieksiene $\mathrm{K}$, et al. ERS guidelines on the diagnosis and treatment of chronic cough in adults and children. Eur Respir J 2020; 55: 1901136.

3 Morice A, Fontana G, Belvisi M, et al. ERS guidelines on the assessment of cough. Eur Respir J 2007; 29: $1256-1276$.

4 Woolf C, Rosenberg A. Objective assessment of cough suppressants under clinical conditions using a tape recorder system. Thorax 1964; 19: 125-130.

5 Birring SS, Fleming T, Matos S, et al. The Leicester Cough Monitor: preliminary validation of an automated cough detection system in chronic cough. Eur Respir J 2008; 31: 1013-1018.

6 Smith JA, Holt K, Dockry R, et al. Performance of a digital signal processing algorithm for the accurate quantification of cough frequency. Eur Respir J 2021; 58: 2004271.

7 Muccino DR, Morice AH, Birring SS, et al. Design and rationale of two phase 3 randomised controlled trials (COUGH-1 and COUGH-2) of gefapixant, a P2X3 receptor antagonist, in refractory or unexplained chronic cough. ERJ Open Res 2020; 6: 00284-2020.

8 Ludbrook VJ, Hanrott KE, Kreindler JL, et al. Adaptive study design to assess effect of TRPV4 inhibition in patients with chronic cough. ERJ Open Res 2021; 7: 00269-2021.

9 Vertigan AE, Kapela SL, Birring SS, et al. Feasibility and clinical utility of ambulatory cough monitoring in an outpatient clinical setting: a real-world retrospective evaluation. ERJ Open Res 2021; 7: 00319-2021.

10 Crooks MG, den Brinker A, Hayman Y, et al. Continuous cough monitoring using ambient sound recording during convalescence from a COPD exacerbation. Lung 2017; 195: 289-294.

11 McGarvey L, Birring S, Morice A, et al. Late breaking abstract - two phase 3 randomized clinical trials of Gefapixant, a P2X3 receptor antagonist, in refractory or unexplained chronic cough (COUGH-1 and COUGH-2). Eur Respir J 2020; 56: Suppl. 64, 3800.

12 Morice $A H$, Jakes AD, Faruqi S, et al. A worldwide survey of chronic cough: a manifestation of enhanced somatosensory response. Eur Respir J 2014; 44: 1149-1155.

13 Morice AH. On chronic cough diagnosis, classification, and treatment. Lung 2021; 199: 433-434. 